\title{
Article
}

\section{Linear differential equations with fast growing coefficients in the unit disc}

\author{
Benharrat Belaïdi ${ }^{1, *}$ and Mohamed Amine Zemirni ${ }^{1}$ \\ 1 Department of Mathematics, Laboratory of Pure and Applied Mathematics, University of Mostaganem (UMAB), B. \\ P. 227 Mostaganem, Algeria. \\ * Correspondence: benharrat.belaidi@univ-mosta.dz
}

Received: 12 February 2020; Accepted: 8 April 2020; Published: 10 April 2020.

Abstract: In this article, we give new conditions on the fast growing analytic coefficients of linear complex differential equations to estimate the iterated $p$-order and iterated $p$-type of all solutions in the unit disc $\mathbb{D}$, where $p \in \mathbb{N} \backslash\{1\}$.

Keywords: Complex differential equation, iterated $p$-order, iterated $p$-type.

MSC: 34M10, 30D35.

\section{Introduction}

onsider the linear differential equation

$$
f^{(k)}+a_{k-1}(z) f^{(k-1)}+\cdots+a_{1}(z) f^{\prime}+a_{0}(z) f=0,
$$

where $k \geq 1$ is an integer, $a_{0}(z), a_{1}(z), \ldots, a_{k-1}(z)$ are analytic functions in the unit disc $\mathbb{D}=\{z \in \mathbb{C}:|z|<1\}$ and $a_{0}(z) \not \equiv 0$. The theory of complex differential equations in the unit disc has been developed since 1980's, see [1]. In the year 2000, Heittokangas in [2] firstly investigated the growth and oscillation theory of Equation (1) when the coefficients $a_{0}(z), a_{1}(z), \ldots, a_{k-1}(z)$ are analytic functions in the unit disc $\mathbb{D}$ by introducing the definition of the function spaces. His results also gave some important tools for further investigations on the theory of meromorphic solutions of Equation (1).

In this article, we investigate the growth of solutions of the Equation (1) when the coefficients $a_{0}(z), a_{1}(z), \ldots, a_{k-1}(z)$ are analytic in $\mathbb{D}$, and we deal with the case that the coefficients are fast growing in $\mathbb{D}$. To define the order of fast-growth of analytic functions, we define inductively for $r \in[0,+\infty), \exp _{0} r=r$, $\exp _{1} r=e^{r}$ and $\exp _{n+1} r=\exp \left(\exp _{n} r\right), n \in \mathbb{N}$. For all $r$ sufficiently large, we define $\log _{0} r=r, \log _{1} r=\log r$ and $\log _{n+1} r=\log \left(\log _{n} r\right), n \in \mathbb{N}$. Also, we need to be familiar with the fundamental results and the standard notations of the Nevanlinna's theory on the complex plane $\mathbb{C}$ and in the unit disc $\mathbb{D}$, for more details on Nevanlinna theory and its applications in complex differential equations in complex plane and in unit disc, we refer to [2-7].

Before stating our main results, we recall definitions and preliminary remarks concerning meromorphic and analytic functions in $\mathbb{D}$. For the definitions and more discussions, we refer the reader to [7-10].

Let $p \geq 1$ be an integer and $f$ be a meromorphic function in $\mathbb{D}$. Then, the iterated $p$-order of $f$ is defined by

$$
\rho_{p}(f)=\limsup _{r \rightarrow 1^{-}} \frac{\log _{p}^{+} T(r, f)}{\log \frac{1}{1-r}}
$$

where $\log _{1}^{+} r=\log ^{+} r=\max \{\log r ; 0\}, \log _{p+1}^{+} r=\log ^{+}\left(\log _{p}^{+} r\right)$ and $T(r, f)$ is the Nevanlinna characteristic function. If $f$ is analytic in $\mathbb{D}$, then the iterated $p$-order of $f$ is defined by

$$
\rho_{M, p}(f)=\limsup _{r \rightarrow 1^{-}} \frac{\log _{p+1}^{+} M(r, f)}{\log \frac{1}{1-r}},
$$


where $M(r, f)=\max \{|f(z)|:|z|=r\}$.

Remark 1. For $p=1, \rho_{1}(f)$ is called order, see [2]. And for $p=2, \rho_{2}(f)$ is called hyper-order, see [11].

Remark 2. It follows by [7, page 205] that if $f$ is an analytic function in $\mathbb{D}$, then we have the inequalities

$$
\rho_{1}(f) \leq \rho_{M, 1}(f) \leq \rho_{1}(f)+1
$$

which are the best possible in the sense that there are analytic functions $g$ and $h$ such that $\rho_{1}(g)=\rho_{M, 1}(g)$ and $\rho_{M, 1}(h)=\rho_{1}(h)+1$, see [12]. However, it follows by [4, Proposition 2.2.2] that $\rho_{p}(f)=\rho_{M, p}(f)$ for $p \geq 2$.

The iterated $p$-type of a meromorphic function $f$ in $\mathbb{D}$ with $0<\rho_{p}(f)<+\infty$ is defined by

$$
\tau_{p}(f)=\limsup _{r \rightarrow 1^{-}}(1-r)^{\rho_{p}(f)} \log _{p-1}^{+} T(r, f),
$$

and if $f$ is analytic in $\mathbb{D}$ with $0<\rho_{M, p}(f)<+\infty$, then the iterated $p$-type is defined by

$$
\tau_{M, p}(f)=\limsup _{r \rightarrow 1^{-}}(1-r)^{\rho_{M, p}(f)} \log _{p}^{+} M(r, f) .
$$

Remark 3. It follows by [4, Proposition 2.2.2] that $\tau_{p}(f)=\tau_{M, p}(f)$ for $p \geq 2$.

\section{Basic results}

Heittokangas et al. in [10] proved the following results.

Theorem 1 ([10]). Let $k \in \mathbb{N}$. If the coefficients $a_{0}(z), a_{1}(z), \ldots, a_{k-1}(z)$ are analytic in $\mathbb{D}$ such that $\rho_{M, p}\left(a_{j}\right)<$ $\rho_{M, p}\left(a_{0}\right)$ for all $j=1, \ldots, k-1$, then all solutions $f \neq \equiv 0$ of $(1)$ satisfy $\rho_{M, p+1}(f)=\rho_{M, p}\left(a_{0}\right)$.

Theorem 2 ([10]). Let $k \in \mathbb{N}$. If the coefficients $a_{0}(z), a_{1}(z), \ldots, a_{k-1}(z)$ are analytic in $\mathbb{D}$ such that $\rho_{M, p}\left(a_{j}\right) \leq$ $\rho_{M, p}\left(a_{0}\right)$ for all $j=1, \ldots, k-1$ and

$$
\sum_{\rho_{M, p}\left(a_{j}\right)=\rho_{M, p}\left(a_{0}\right)} \tau_{M, p}\left(a_{j}\right)<\tau_{M, p}\left(a_{0}\right),
$$

then all solutions $f \not \equiv 0$ of $(1)$ satisfy $\rho_{M, p+1}(f)=\rho_{M, p}\left(a_{0}\right)$.

Hamouda in [13], gave an improvement of Theorem 2 as follows.

Theorem 3 ([13]). Let $k \in \mathbb{N}$. If the coefficients $a_{0}(z), a_{1}(z), \ldots, a_{k-1}(z)$ are analytic in $\mathbb{D}$ such that $\rho_{M, p}\left(a_{j}\right) \leq$ $\rho_{M, p}\left(a_{0}\right)$ for all $j=1, \ldots, k-1$ and

$$
\max \left\{\tau_{M, p}\left(a_{j}\right): \rho_{M, p}\left(a_{j}\right)=\rho_{M, p}\left(a_{0}\right)\right\}<\tau_{M, p}\left(a_{0}\right)
$$

then all solutions $f \not \equiv 0$ of $(1)$ satisfy $\rho_{M, p+1}(f)=\rho_{M, p}\left(a_{0}\right)$.

Our proofs depend mainly upon the following lemmas. Before starting these lemmas, we recall the concept of logarithmic measure. The logarithmic measure of a set $S \subset(0,1)$ is given by

$$
\operatorname{lm}(S):=\int_{S} \frac{d t}{1-t}
$$

The set $F \subset[0,1)$ in all this paper is not necessarily the same at each occurrence, but it is always of finite logarithmic measure, that is $\operatorname{lm}(F)<+\infty$.

To avoid some problems of the exceptional sets, we need the following lemma. 
Lemma 1 ([2,14]). Let $g:[0,1) \mapsto \mathbb{R}$ and $h:[0,1) \mapsto \mathbb{R}$ be monotone non-decreasing functions such that $g(r) \leq h(r)$ holds outside of an exceptional set $F \subset[0,1)$ of finite logarithmic measure. Then there exists a $d \in(0,1)$ such that if $s(r)=1-d(1-r)$, then $g(r) \leq h(s(r))$ for all $r \in[0,1)$.

Lemma 2 ([12, Theorem 3.1]). Let $k$ and $j$ be integers satisfying $k>j \geq 0$, and let $\varepsilon>0$ and $d \in(0,1)$. If $f$ is a meromorphic in $\mathbb{D}$ such that $f^{(j)} \not \equiv 0$, then

$$
\left|\frac{f^{(k)}(z)}{f^{(j)}(z)}\right| \leq\left[\left(\frac{1}{1-|z|}\right)^{2+\varepsilon} \max \left\{\log \frac{1}{1-|z|} ; T(s(|z|), f)\right\}\right]^{k-j}
$$

for $|z| \notin F$, where $F \subset[0,1)$ is a set of finite logarithmic measure, and where $s(|z|)=1-d(1-r)$.

Lemma 3 ([10]). Let $f$ be a meromorphic function in the unit disc with $\rho_{p}(f):=\rho<+\infty$ for some $p \in \mathbb{N}$, and let $\varepsilon>0$ be a given constant. Then, there exists a set $F \subset(0,1)$ of finite logarithmic measure such that for all $z$ with $|z|=r \notin F$ and for all integer $j \geq 1$, we have:

(i) If $p=1$, then

$$
\left|\frac{f^{(j)}(z)}{f(z)}\right| \leq \frac{1}{(1-r)^{j(\rho+2+\varepsilon)}}
$$

(ii) If $p \geq 2$, then

$$
\left|\frac{f^{(j)}(z)}{f(z)}\right| \leq \exp _{p-1}\left\{\frac{1}{(1-r)^{\rho+\varepsilon}}\right\}
$$

Lemma 4 ([10]). Let $a_{0}(z), a_{1}(z), \ldots, a_{k-1}(z)$ be analytic functions in the unit disc $\mathbb{D}$. Then, every solution $f \not \equiv 0$ of the Equation (1) satisfies

$$
\rho_{p+1}(f)=\rho_{M, p+1}(f) \leq \max \left\{\rho_{M, p}\left(a_{j}\right): j=0, \ldots, k-1\right\} .
$$

Remark 4. If $p \geq 2$, then by Remark 2 and Lemma 4 , we obtain that very solution $f \not \equiv 0$ of the Equation (1) satisfies

$$
\rho_{p+1}(f) \leq \max \left\{\rho_{p}\left(a_{j}\right): j=0, \ldots, k-1\right\} .
$$

Lemma $5([2,3,7])$. Let $f$ be a meromorphic function in the unit disc $\mathbb{D}$ and let $k \in \mathbb{N}$. Then

$$
m\left(r, \frac{f^{(k)}}{f}\right)=S(r, f)
$$

where $S(r, f)=O\left(\log ^{+} T(r, f)+\log \left(\frac{1}{1-r}\right)\right)$, possibly outside a set $F \subset[0,1)$ with finite logarithmic measure.

Lemma 6 ([15]). Let $f$ be a meromorphic function in the unit disc $\mathbb{D}$ for which $i(f)=p \geq 1$ and $\rho_{p}(f)=\rho<+\infty$, and let $k \geq 1$ be an integer. Then for any $\varepsilon>0$,

$$
m\left(r, \frac{f^{(k)}}{f}\right)=O\left(\exp _{p-2}\left\{\frac{1}{1-r}\right\}^{\rho+\varepsilon}\right)
$$

holds for all $r$ outside a set $F \subset[0,1)$ with $\int_{F} \frac{d r}{1-r}<+\infty$.

Lemma 7. For an integer $p \geq 2$, let $f$ be a meromorphic function in $\mathbb{D}$ such that $0<\rho_{p}(f)=\rho<+\infty, 0<\tau_{p}(f)=$ $\tau<+\infty$ and $0<\tau_{p}^{*}(f)=\tau^{*}<+\infty$ (see Definition 1). Then for any given $\eta<\tau^{*}$, there exists a subset $E \subset[0,1)$ that has an infinite logarithmic measure $\int_{E} \frac{d r}{1-r}=+\infty$ such that for all $r \in E$, we have

$$
\log _{p-2} T(r, f)>\eta \exp \left\{\frac{\tau}{(1-r)^{\rho}}\right\} \text {. }
$$


Proof. By the definition of $\tau_{p}^{*}(f)$, there exists an increasing sequence $\left\{r_{m}\right\}_{m=1}^{+\infty} \subset[0,1)$ satisfying $\frac{1}{m}+$ $\left(1-\frac{1}{m}\right) r_{m}<r_{m+1},\left(r_{m} \longrightarrow 1^{-}, m \longrightarrow+\infty\right)$ and

$$
\lim _{m \rightarrow+\infty} \frac{\log _{p-2} T\left(r_{m}, f\right)}{\exp \left\{\frac{\tau}{\left(1-r_{m}\right)^{p}}\right\}}=\tau^{*}
$$

Then, for any given $0<\varepsilon<\tau^{*}$, there exists a positive integer $m_{0}$ such that for all $m \geq m_{0}$, we have

$$
\log _{p-2} T\left(r_{m}, f\right)>\left(\tau^{*}-\varepsilon\right) \exp \left\{\frac{\tau}{\left(1-r_{m}\right)^{\rho}}\right\} \text {. }
$$

For $r \in\left[r_{m}, \frac{1}{m}+\left(1-\frac{1}{m}\right) r_{m}\right]$, we get

$$
\lim _{m \rightarrow+\infty} \frac{\exp \left\{\tau\left[\left(1-\frac{1}{m}\right)\left(\frac{1}{1-r}\right)\right]^{\rho}\right\}}{\exp \left\{\frac{\tau}{(1-r)^{\rho}}\right\}}=1
$$

Then for any given $0<\eta<\tau^{*}-\varepsilon$, there exists a positive integer $m_{1}$ such that for all $m \geq m_{1}$, and for all $r \in\left[r_{m}, \frac{1}{m}+\left(1-\frac{1}{m}\right) r_{m}\right]$, we have

$$
\frac{\exp \left\{\tau\left[\left(1-\frac{1}{m}\right)\left(\frac{1}{1-r}\right)\right]^{\rho}\right\}}{\exp \left\{\frac{\tau}{(1-r)^{\rho}}\right\}}>\frac{\eta}{\tau^{*}-\varepsilon} .
$$

By (4) and (5), for all $m \geq m_{2}=\max \left\{m_{0} ; m_{1}\right\}$ and for all $r \in\left[r_{m}, \frac{1}{m}+\left(1-\frac{1}{m}\right) r_{m}\right]$, we have

$$
\begin{aligned}
\log _{p-2} T(r, f) & \geq \log _{p-2}^{+} T\left(r_{m}, f\right)>\left(\tau^{*}-\varepsilon\right) \exp \left\{\frac{\tau}{\left(1-r_{m}\right)^{\rho}}\right\} \\
& \geq\left(\tau^{*}-\varepsilon\right) \exp \left\{\tau\left[\left(1-\frac{1}{m}\right)\left(\frac{1}{1-r}\right)\right]^{\rho}\right\}>\eta \exp \left\{\frac{\tau}{(1-r)^{\rho}}\right\} .
\end{aligned}
$$

Set

$$
E=\bigcup_{m=m_{2}}^{+\infty}\left[r_{m}, \frac{1}{m}+\left(1-\frac{1}{m}\right) r_{m}\right]
$$

Then

$$
\int_{E} \frac{d t}{1-t}=\sum_{m=m_{2}}^{+\infty} \int_{r_{m}}^{\frac{1}{m}+\left(1-\frac{1}{m}\right) r_{m}} \frac{d t}{1-t}=\sum_{m=m_{2}}^{+\infty} \log \frac{m}{m-1}=+\infty
$$

By using similar reasoning as in the proof of Lemma 7, we easily obtain the following lemma.

Lemma 8. For an integer $p \geq 2$, let $f$ be a meromorphic function in $\mathbb{D}$ such that $0<\rho_{M, p}(f)=\rho<+\infty, 0<$ $\tau_{M, p}(f)=\tau<+\infty$ and $0<\tau_{M, p}^{*}(f)=\tau^{*}<+\infty$. Then for any given $\eta<\tau^{*}$, there exists a subset $E \subset[0,1)$ that has an infinite logarithmic measure $\int_{E} \frac{d r}{1-r}=+\infty$ such that for all $r \in E$, we have

$$
\log _{p-1} M(r, f)>\eta \exp \left\{\frac{\tau}{(1-r)^{\rho}}\right\} .
$$

Lemma 9 ([16]). Let $f$ be a solution of Equation (1), where the coefficients $a_{j}(z)(j=0, \ldots, k-1)$ are analytic functions in the disc $\Delta_{R}=\{z \in \mathbb{C}:|z|<R\}, 0<R \leq \infty$. Let $n_{c} \in\{1, \ldots, k\}$ be the number of nonzero coefficients $a_{j}(z)$ 
$(j=0, \ldots, k-1)$, and let $\theta \in[0,2 \pi]$ and $\varepsilon>0$. If $z_{\theta}=v e^{i \theta} \in \Delta_{R}$ is such that $a_{j}\left(z_{\theta}\right) \neq 0$ for some $j=0, \ldots, k-1$, then for all $v<r<R$,

$$
\left|f\left(r e^{i \theta}\right)\right| \leq C \exp \left(n_{c} \int_{v}^{r} \max _{j=0, \ldots, k-1}\left|a_{j}\left(t e^{i \theta}\right)\right|^{\frac{1}{k-j}} d t\right)
$$

where $C>0$ is a constant satisfying

$$
C \leq(1+\varepsilon) \max _{j=0, \ldots, k-1}\left(\frac{\left|f^{(j)}\left(z_{\theta}\right)\right|}{\left(n_{\mathcal{c}}\right)^{j} \max _{n=0, \ldots, k-1}\left|a_{n}\left(z_{\theta}\right)\right|^{\frac{j}{k-n}}}\right) .
$$

Lemma 10. Let $\left\{a_{j}(z)\right\}_{0 \leq j \leq k-1}$ be analytic functions in the disc $\mathbb{D}$ such that $0<p<\infty$ and $0<\max \left\{\rho_{M, p}\left(a_{j}\right)\right.$ : $j=1, \ldots, k-1\} \leq \rho_{M, p}\left(a_{0}\right)=\rho<\infty$ and $\max \left\{\tau_{M, p}\left(a_{j}\right): j=1, \ldots, k-1\right\} \leq \tau_{M, p}\left(a_{0}\right)=\tau<\infty$. Then, every solution $f \not \equiv 0$ of the Equation (1) with $\rho_{p+1}(f)=\rho$ satisfies $\tau_{p+1}(f) \leq \tau$.

Proof. Let $f \not \equiv 0$ be a solution of $(1)$ with $\rho_{p+1}(f)=\rho$. Let $\theta_{0} \in[0,2 \pi)$ be such that $\left|f\left(r e^{i \theta_{0}}\right)\right|=M(r, f)$. By Lemma 9, we have

$$
\begin{aligned}
M(r, f) & \leq C \exp \left(n_{\mathcal{c}} \int_{v}^{r} \max _{j=0, \ldots, k-1}\left|a_{j}\left(t e^{i \theta}\right)\right|^{\frac{1}{k-j}} d t\right) \\
& \leq C \exp \left(n_{c} \int_{v}^{r} \max _{j=0, \ldots, k-1}\left(M\left(r, a_{j}\right)\right)^{\frac{1}{k-j}} d t\right) \\
& \leq C \exp \left(n_{\mathcal{c}}(r-v) \max _{j=0, \ldots, k-1}\left\{M\left(r, a_{j}\right)\right\}\right) .
\end{aligned}
$$

We have $\max \left\{\rho_{M, p}\left(a_{j}\right): j=0,1, \ldots, k-1\right\}=\rho_{M, p}\left(a_{0}\right)=\rho$. By the definition of $\tau_{M, p}\left(a_{j}\right)$, for any given $\varepsilon>0$ and $r \rightarrow 1^{-}$, we obtain

$$
M\left(r, a_{j}\right) \leq \exp _{p}\left\{\frac{\tau_{M, p}\left(a_{j}\right)+\frac{\varepsilon}{2}}{(1-r)^{\rho_{M, p}\left(a_{j}\right)}}\right\} \leq \exp _{p}\left\{\frac{\tau+\frac{\varepsilon}{2}}{(1-r)^{\rho}}\right\}(j=0,1, \ldots, k-1) .
$$

By (6) and (7), we have for $r \rightarrow 1^{-}$

$$
M(r, f) \leq \exp _{p+1}\left\{\frac{\tau+\varepsilon}{(1-r)^{\rho}}\right\}
$$

Then, it follows from (8), arbitrariness of $\varepsilon>0$ that $\tau_{p+1}(f)=\tau_{M, p+1}(f) \leq \tau$.

\section{Main results}

In this article, we aim to answer the following questions:

1. What can be said about the growth of solutions of the Equation (1) in the case when $\rho_{M, p}\left(a_{j}\right) \leq \rho_{M, p}\left(a_{0}\right)$ for all $j=1, \ldots, k-1$ and

$$
\max \left\{\tau_{M, p}\left(a_{j}\right): \rho_{M, p}\left(a_{j}\right)=\rho_{M, p}\left(a_{0}\right)\right\} \leq \tau_{M, p}\left(a_{0}\right) ?
$$

2. What happened when we replace $\rho_{M, p}$ and $\tau_{M, p}$ by $\rho_{p}$ and $\tau_{p}$ ?

As the first result, we give an improvement to Theorems 1 and 2 of [13].

Theorem 4. Let $a_{0}(z), a_{1}(z), \ldots, a_{k-1}(z)$ be meromorphic functions in the unit disc $\mathbb{D}$, and $a_{0}(z) \not \equiv 0$. Suppose that there exist a point $\omega \in \partial \mathbb{D}$, a curve $\gamma$ tending to $\omega$ and a set $F_{1} \subset(0,1)$ of finite logarithmic measure such that for $z \in \gamma$ and $|z|=r \notin F_{1}$, we have for the largest integer $p \geq 1$ 


$$
\lim _{z \rightarrow \omega} \frac{\sum_{j=1}^{k-1}\left|a_{j}(z)\right|+1}{\left|a_{0}(z)\right|} \exp _{p}\left\{\frac{\lambda}{(1-r)^{\alpha}}\right\}=0
$$

for all $\lambda>0$ and $\alpha>0$. Then, every nontrivial meromorphic solution $f$ of the Equation (1) satisfies $\rho_{p+1}(f)=+\infty$.

Proof. Suppose that $f \not \equiv 0$ is a solution of the Equation (1) with $\rho_{p+1}(f)=\rho<+\infty$. By (1), $f$ satisfies

$$
1 \leq \frac{1}{\left|a_{0}(z)\right|}\left|\frac{f^{(k)}}{f}\right|+\sum_{j=1}^{k-1} \frac{\left|a_{j}(z)\right|}{\left|a_{0}(z)\right|}\left|\frac{f^{(j)}}{f}\right| .
$$

For $p \geq 1$, by (3), for all $z$ satisfying $|z|=r \notin F$ ( $F$ has finite logarithmic measure), we obtain

$$
\left|\frac{f^{(j)}(z)}{f(z)}\right| \leq \exp _{p}\left\{\frac{1}{(1-|z|)^{\alpha}}\right\},
$$

where $\alpha>0$ is a constant which depends on $\rho, \varepsilon$ and $j=1, \ldots, k$. By substituting (11) into (10), it yields

$$
1 \leq \frac{\sum_{j=1}^{k-1}\left|a_{j}(z)\right|+1}{\left|a_{0}(z)\right|} \exp _{p}\left\{\frac{1}{(1-|z|)^{\alpha}}\right\} .
$$

By (9), for $z \in \gamma$ such that $|z|=r \notin F_{1}$ ( $F_{1}$ has finite logarithmic measure), we know that as $z \rightarrow \omega$

$$
\frac{\sum_{j=1}^{k-1}\left|a_{j}(z)\right|+1}{\left|a_{0}(z)\right|} \exp _{p}\left\{\frac{1}{(1-|z|)^{\alpha}}\right\} \rightarrow 0 .
$$

Thus, for all $z \in \gamma$ with $|z|=r \notin F_{1} \cup F$, by (12) and (13), we get a contradiction. Hence, every meromorphic solution $f \not \equiv 0$ of (1) has an infinite $(p+1)$-order.

Remark 5. In [13], under the same hypotheses of Theorem 4, Hamouda obtained that $\rho_{p+1}(f) \geq \alpha$.

In all the next, we consider $p \in \mathbb{N} \backslash\{1\}$. In trying to give an answer on the above questions, we prove the following results.

Theorem 5. Let $a_{0}(z), a_{1}(z), \ldots, a_{k-1}(z)$ be analytic functions in the unit disc $\mathbb{D}$ satisfying $\rho_{M, p}\left(a_{j}\right) \leq \rho_{M, p}\left(a_{0}\right)=\rho$ $(0<\rho<+\infty)$ and $\tau_{M, p}\left(a_{j}\right) \leq \tau_{M, p}\left(a_{0}\right)=\tau(0<\tau<+\infty)$ for all $j=1, \ldots, k-1$. Suppose that there exist two positive real numbers $\alpha$ and $\beta$ with $0 \leq \beta<\alpha$, such that

$$
\left|a_{0}(z)\right| \geq \exp _{p-1}\left\{\alpha \exp \frac{\tau}{(1-r)^{\rho}}\right\}
$$

and

$$
\left|a_{j}(z)\right| \leq \exp _{p-1}\left\{\beta \exp \frac{\tau}{(1-r)^{\rho}}\right\}, j=1, \ldots, k-1
$$

as $|z|=r \rightarrow 1^{-}$for $r \in E_{1}$ ( $E_{1}$ is of infinite logarithmic measure). Then, every solution $f \not \equiv 0$ of the Equation (1) satisfies $\rho_{p}(f)=+\infty, \rho_{p+1}(f)=\rho$ and $d^{\rho} \tau \leq \tau_{p+1}(f) \leq \tau, d \in(0,1)$.

Proof. Let $f \not \equiv 0$ be a solution of the Equation (1). By (1), $f$ satisfies

$$
\left|a_{0}(z)\right| \leq\left|\frac{f^{(k)}}{f}\right|+\sum_{j=1}^{k-1}\left|a_{j}(z)\right| \frac{f^{(j)}}{f} \mid .
$$


By hypotheses of Theorem 5 and Lemma 4 , we know that $\rho_{p+1}(f) \leq \rho$. Suppose that $\rho_{p+1}(f)=\rho_{1}<\rho$. Then, by (3) for all $0<\varepsilon<\rho-\rho_{1}$, we have

$$
\left|\frac{f^{(j)}(z)}{f(z)}\right| \leq \exp _{p}\left\{\frac{1}{(1-r)^{\rho_{1}+\varepsilon}}\right\}, j=1, \ldots, k,
$$

where $|z|=r \notin F$. By substituting (14), (15) and (17) into (16) we obtain

$$
\exp _{p-1}\left\{\alpha \exp \frac{\tau}{(1-r)^{\rho}}\right\} \leq k \exp _{p-1}\left\{\beta \exp \frac{\tau}{(1-r)^{\rho}}\right\} \exp _{p}\left\{\frac{1}{(1-r)^{\rho_{1}+\varepsilon}}\right\}
$$

for all $r \in E_{1} \backslash F$. Hence, we get

$$
(\alpha-\beta) \exp \left\{\frac{\tau}{(1-r)^{\rho}}\right\} \leq \exp \left\{\frac{1}{(1-r)^{\rho_{1}+\varepsilon}}\right\}+C_{1}
$$

for some constant $C_{1}>0$, which is a contradiction as $|z|=r \rightarrow 1^{-}, r \in E_{1} \backslash F$, since $\alpha>\beta \geq 0$ and $\rho>\rho_{1}+\varepsilon$. Thus, $\rho_{p+1}(f)=\rho$.

Now, by Lemma 2, we have for $j=1, \ldots, k$

$$
\left|\frac{f^{(j)}(z)}{f(z)}\right| \leq\left[\left(\frac{1}{1-|z|}\right)^{2+\varepsilon} \max \left\{\log \frac{1}{1-|z|} ; T(s(|z|), f)\right\}\right]^{k}
$$

for all $r \notin F_{2} \cup[0,1]$. From, (14), (15), (16) and (20), we obtain

$$
\exp _{p-1}\left\{\alpha \exp \frac{\tau}{(1-r)^{\rho}}\right\} \leq k \exp _{p-1}\left\{\beta \exp \frac{\tau}{(1-r)^{\rho}}\right\}\left(\frac{1}{1-r}\right)^{(2+\varepsilon) k} T^{k}(s(r), f)
$$

for all $r \in E_{1} \backslash\left(F_{2} \cup[0,1]\right)$. Hence

$$
\log (\alpha-\beta)+\frac{\tau}{(1-r)^{\rho}} \leq \log _{p} \frac{1}{1-r}+\log _{p} T(s(r), f)+C_{2}
$$

for some constant $C_{2}>0$ and for all $r \in E_{1} \backslash\left(F_{2} \cup[0,1]\right)$. Setting $R=s(r)=1-d(1-r), d \in(0,1)$. We have $1-r=\frac{1-R}{d}, d \in(0,1)$. Then by Lemma 1 , we obtain we obtain for $R \longrightarrow 1^{-}$

$$
\log (\alpha-\beta)+\frac{d^{\rho} \tau}{(1-R)^{\rho}} \leq \log _{p} \frac{d}{1-R}+\log _{p} T(R, f)+C_{2}
$$

Since $0<\rho_{p+1}(f)=\rho<\infty$, from (23) we deduce that

$$
\tau_{p+1}(f)=\limsup _{R \rightarrow 1^{-}} \frac{\log _{p}^{+} T(R, f)}{\frac{1}{(1-R)^{\rho}}} \geq d^{\rho} \tau .
$$

By Lemma 10, we conclude that $d^{\rho} \tau \leq \tau_{p+1}(f) \leq \tau$.

Theorem 6. Let $a_{0}(z), a_{1}(z), \ldots, a_{k-1}(z)$ be analytic functions in the unit disc $\mathbb{D}$ satisfying $\rho_{p}\left(a_{j}\right) \leq \rho_{p}\left(a_{0}\right)=\rho$ $(0<\rho<+\infty)$ and $\tau_{p}\left(a_{j}\right) \leq \tau_{p}\left(a_{0}\right)=\tau(0<\tau<+\infty)$ for all $j=1, \ldots, k-1$. Suppose that there exist two positive real numbers $\alpha$ and $\beta$ with $0 \leq \beta<\alpha$, such that

$$
m\left(r, a_{0}\right) \geq \exp _{p-2}\left\{\alpha \exp \frac{\tau}{(1-r)^{\rho}}\right\}
$$

and

$$
m\left(r, a_{j}\right) \leq \exp _{p-2}\left\{\beta \exp \frac{\tau}{(1-r)^{\rho}}\right\}, j=1, \ldots, k-1
$$


as $|z|=r \rightarrow 1^{-}$for $r \in E_{2}$ ( $E_{2}$ is of infinite logarithmic measure). Then, every solution $f \not \equiv 0$ of the Equation (1) satisfies $\rho_{p}(f)=+\infty, \rho_{p+1}(f)=\rho$ and $d^{\rho} \tau \leq \tau_{p+1}(f) \leq \tau, d \in(0,1)$.

Proof. Let $f \not \equiv 0$ be a solution of the Equation (1). By (1) we can write

$$
a_{0}(z)=-\left(\frac{f^{(k)}}{f}+\sum_{j=1}^{k-1} a_{j}(z) \frac{f^{(j)}}{f}\right) .
$$

By hypotheses of Theorem 6 and Lemma 4 , we know that $\rho_{p+1}(f) \leq \rho$. Suppose that $\rho_{p+1}(f)=\rho_{1}<\rho$. Then by Lemma 6, for all $0<\varepsilon<\rho-\rho_{1}$ and for all $|z|=r \notin F$, we have for $j=1, \ldots, k$

$$
m\left(r, \frac{f^{(j)}}{f}\right)=O\left(\exp _{p-1}\left\{\frac{1}{(1-r)^{\rho_{1}+\varepsilon}}\right\}\right) .
$$

Now, let $p \geq 2$, it follows by (24), (25), (26) and (27) that

$$
\begin{aligned}
\exp _{p-2}\left\{\alpha \exp \frac{\tau}{(1-r)^{\rho}}\right\} & \leq m\left(r, a_{0}\right) \\
& \leq \sum_{j=1}^{k-1} m\left(r, a_{j}\right)+\sum_{j=1}^{k-1} m\left(r, \frac{f^{(j)}}{f}\right)+O(1) \\
& \leq(k-1) \exp _{p-2}\left\{\beta \exp \frac{\tau}{(1-r)^{\rho}}\right\}+M \exp _{p-1}\left\{\frac{1}{(1-r)^{\rho_{1}+\varepsilon}}\right\}
\end{aligned}
$$

holds for all $z$ satisfying $|z|=r \in E_{2} \backslash F$ as $r \rightarrow+\infty$, and $M>0$ is some constant. Hence, from (28) we obtain

$$
(\alpha-\beta) \exp \left\{\frac{\tau}{(1-r)^{\rho}}\right\} \leq \exp \left\{\frac{1}{(1-r)^{\rho_{1}+\varepsilon}}\right\}+C_{3}
$$

for some constant $C_{3}>0$, which is a contradiction as $|z|=r \rightarrow 1^{-}, r \in E_{2} \backslash F$, since $\alpha>\beta \geq 0$ and $\rho>\rho_{1}+\varepsilon$. Thus, $\rho_{p+1}(f)=\rho$.

Now, it follows by (24), (25), (26) and Lemma 5 that

$$
\begin{aligned}
\exp _{p-2}\left\{\alpha \exp \frac{\tau}{(1-r)^{\rho}}\right\} & \leq m\left(r, a_{0}\right) \\
& \leq \sum_{j=1}^{k-1} m\left(r, a_{j}\right)+\sum_{j=1}^{k-1} m\left(r, \frac{f^{(j)}}{f}\right)+O(1) \\
& \leq(k-1) \exp _{p-2}\left\{\beta \exp \frac{\tau}{(1-r)^{\rho}}\right\}+O\left(\log ^{+} T(r, f)+\log \left(\frac{1}{1-r}\right)\right)
\end{aligned}
$$

for all sufficiently large $|z|=r \in E_{2} \backslash F$. Then, for all sufficiently large $|z|=r \in E_{2} \backslash F$

$$
\log (\alpha-\beta)+\frac{\tau}{(1-r)^{\rho}} \leq \log _{p}^{+} T(r, f)+\log _{p}\left(\frac{1}{1-r}\right)+C_{4}
$$

for some constant $C_{4}>0$. Then by Lemma 1, we obtain for $|z|=r \in E_{2}, s(r) \rightarrow 1^{-}$

$$
\log (\alpha-\beta)+\frac{\tau}{(1-r)^{\rho}} \leq \log _{p}^{+} T(s(r), f)+\log _{p}\left(\frac{1}{1-s(r)}\right)+C_{4}
$$

where $s(r)=1-d(1-r), d \in(0,1)$. Hence, by (31) we obtain

$$
\tau_{p+1}(f)=\limsup _{s(r) \rightarrow 1^{-}} \frac{\log _{p}^{+} T(s(r), f)}{\frac{1}{(1-s(r))^{\rho}}} \geq d^{\rho} \tau .
$$

By Lemma 10, we conclude that $d^{\rho} \tau \leq \tau_{p+1}(f) \leq \tau$. 
Hamouda in [17], to study the growth of meromorphic solutions of differential equations with finite iterated $p$-order in complex plane, introduced new type of growth (see [17, p. 46]). According to the definition of this new type of growth, we introduce a new definition of type of growth that we note $\tau_{p}^{*}(f)$ related to iterated $p$-order of meromorphic function $f$ in the unit disc, as follows.

Definition 1. For $p \geq 2$, let $f$ be a meromorphic function of finite iterated $p$-order in $\mathbb{D}$ such that $0<\rho_{p}(f)=$ $\rho<+\infty$ and $0<\tau_{p}(f)=\tau<+\infty$, we define $\tau_{p}^{*}(f)$ by

$$
\tau_{p}^{*}(f)=\limsup _{r \rightarrow 1^{-}} \frac{\log _{p-2}^{+} T(r, f)}{\exp \left\{\frac{\tau}{(1-r)^{\rho}}\right\}} .
$$

If $f$ is an analytic function in $\mathbb{D}$ with $0<\tau_{M, p}(f)=\tau_{M}<+\infty$, we also define

$$
\tau_{M, p}^{*}(f)=\limsup _{r \rightarrow 1^{-}} \frac{\log _{p-1}^{+} M(r, f)}{\exp \left\{\frac{\tau_{M}}{(1-r)^{\rho}}\right\}}
$$

The following theorems improves and extends Theorems 2 and 3.

Theorem 7. Let $a_{0}(z), a_{1}(z), \ldots, a_{k-1}(z)$ be analytic functions in the unit disc $\mathbb{D}$ satisfying $\rho_{M, p}\left(a_{j}\right) \leq \rho_{M, p}\left(a_{0}\right)=\rho$ $(0<\rho<+\infty)$ and $\tau_{M, p}\left(a_{j}\right) \leq \tau_{M, p}\left(a_{0}\right)=\tau(0<\tau<+\infty)$ for all $j=1, \ldots, k-1$ and

$$
\max \left\{\tau_{M, p}^{*}\left(a_{j}\right): j=1, \ldots, k-1\right\}<\tau_{M, p}^{*}\left(a_{0}\right) .
$$

Then all solutions $f \not \equiv 0$ of $(1)$ satisfy $\rho_{p}(f)=+\infty, \rho_{p+1}(f)=\rho$ and $d^{\rho} \tau \leq \tau_{p+1}(f) \leq \tau, d \in(0,1)$.

Proof. Suppose that all coefficients of the Equation (1) satisfy the hypotheses of Theorem 7. Now, let $\alpha$ and $\beta$ be two real numbers such that

$$
\max \left\{\tau_{M, p}^{*}\left(a_{j}\right): j=1, \ldots, k-1\right\}<\beta<\alpha<\tau_{M, p}^{*}\left(a_{0}\right) .
$$

Because all coefficients are analytic, then for $r \longrightarrow 1^{-}$

$$
\left|a_{j}(z)\right| \leq \exp _{p-1}\left\{\beta \exp \frac{\tau}{(1-r)^{\rho}}\right\}, \quad j=1, \ldots, k-1
$$

and by Lemma 8 , we have

$$
M\left(r, a_{0}\right)=\left|a_{0}(z)\right|>\exp _{p-1}\left\{\alpha \exp \frac{\tau}{(1-r)^{\rho}}\right\}
$$

for all $r \in E$ ( $E$ is a set of infinite logarithmic measure). From (32) and (33), and by Theorem 5, we obtain the result.

Theorem 8. Let $a_{0}(z), a_{1}(z), \ldots, a_{k-1}(z)$ be analytic functions in the unit disc $\mathbb{D}$ satisfying $\rho_{p}\left(a_{j}\right) \leq \rho_{p}\left(a_{0}\right)=\rho$ $(0<\rho<+\infty), \tau_{p}\left(a_{j}\right) \leq \tau_{p}\left(a_{0}\right)=\tau(0<\tau<+\infty)$ for all $j=1, \ldots, k-1$ and

$$
\max \left\{\tau_{p}^{*}\left(a_{j}\right): j=1, \ldots, k-1\right\}<\tau_{p}^{*}\left(a_{0}\right) .
$$

Then all solutions $f \not \equiv 0$ of $(1)$ satisfy $\rho_{p}(f)=+\infty, \rho_{p+1}(f)=\rho$ and $d^{\rho} \tau \leq \tau_{p+1}(f) \leq \tau, d \in(0,1)$.

Proof. Suppose that all coefficients of the Equation (1) satisfy the hypotheses of Theorem 8 . Now, let $\alpha$ and $\beta$ be two real numbers such that

$$
\max \left\{\tau_{p}^{*}\left(a_{j}\right): j=1, \ldots, k-1\right\}<\beta<\alpha<\tau_{p}^{*}\left(a_{0}\right) .
$$


Since all coefficients are analytic, then for $r \longrightarrow 1^{-}$

$$
m\left(r, a_{j}\right) \leq \exp _{p-2}\left\{\beta \exp \frac{\tau}{(1-r)^{\rho}}\right\}, \quad j=1, \ldots, k-1
$$

and by Lemma 7, we have

$$
T\left(r, a_{0}\right)=m\left(r, a_{0}\right)>\exp _{p-2}\left\{\alpha \exp \frac{\tau}{(1-r)^{\rho}}\right\}
$$

for all $r \in E$ ( $E$ is a set of infinite logarithmic measure). From (34) and (35), and by Theorem 6, we obtain the result.

For some related results in the whole complex plane, see [18].

Acknowledgments: The authors would like to thank the referee for his/her valuables remarks, which led to an improvement of the presentation of this paper. This paper is supported by University of Mostaganem (UMAB) (PRFU Project Code C00L03UN270120180005).

Author Contributions: All authors contributed equally to the writing of this paper. All authors read and approved the final manuscript.

Conflicts of Interest: "The authors declare no conflict of interest."

\section{References}

[1] Pommerenke, C. (1982). On the mean growth of the solutions of complex linear differential equations in the disk. Complex Variables and Elliptic Equations, 1(1), 23-38.

[2] Heittokangas, J. (2000). On complex differential equations in the unit disc (Vol. 122). Suomalainen Tiedeakatemia.

[3] Hayman, W. K. (1964). Meromorphic functions, Oxford mathematical monographs.

[4] Laine, I. (1993). Nevanlinna theory and complex differential equations (Vol. 15). Walter de Gruyter \& Co., Berlin.

[5] Laine, I. (2008). Complex differential equations, Handbook of differential equations: ordinary differential equations. Vol. IV, 269-363, Handb. Differ. Equ., Elsevier/North-Holland, Amsterdam.

[6] Yang, C. C., \& Yi, H. X. (2003). Uniqueness theory of meromorphic functions (Vol. 557). Springer Science \& Business Media.

[7] Tsuji, M. (1975). Potential theory in modern function theory Chelsea Publishing Co., New York.

[8] Cao, T. B., \& Yi, H. X. (2006). The growth of solutions of linear differential equations with coefficients of iterated order in the unit disc. Journal of Mathematical Analysis and Applications, 319(1), 278-294.

[9] Cao, T. B. (2009). The growth, oscillation and fixed points of solutions of complex linear differential equations in the unit disc. Journal of Mathematical Analysis and Applications, 352(2), 739-748.

[10] Heittokangas, J., Korhonen, R., \& Rättyä, J. (2006). Fast growing solutions of linear differential equations in the unit disc. Results in Mathematics, 49(3-4), 265-278.

[11] Li, Y. Z. (2002). On the growth of the solution of two-order differential equations in the unit disc. Pure and Applied Mathematics, 4, 295-300.

[12] Chyzhykov, I., Gundersen, G. G., \& Heittokangas, J. (2003). Linear differential equations and logarithmic derivative estimates. Proceedings of the London Mathematical Society, 86(3), 735-754.

[13] Hamouda, S. (2013). Iterated order of solutions of linear differential equations in the unit disc. Computational Methods and Function Theory, 13(4), 545-555.

[14] Bank, S. B. (1972). A general theorem concerning the growth of solutions of first-order algebraic differential equations. Compositio Mathematica, 25(1), 61-70.

[15] Belaidi, B. (2010). Oscillation of fast growing solutions of linear differential equations in the unit disc. Acta Universitatis Sapientiae, 2 (1), 25-38.

[16] Heittokangas, J., Korhonen, R., \& Rattya, J.(2004). Growth estimates for solutions of linear complex differential equations, Annales Academiae Scientiarum Fennicae Mathematica, 29, 233-246.

[17] Hamouda, S. (2015). On the iterated order of solutions of linear differential equations in the complex plane. Southeast Asian Bulletin of Mathematics, 39(1), 45-55.

[18] Zemirni, M. A., \& Belaidi, B. (2018). Linear differential equations with fast-growing coefficients in complex plane. Nonlinear Studies, 25(3), 719-731. 
(C) 2020 by the authors; licensee PSRP, Lahore, Pakistan. This article is an open access article distributed under the terms and conditions of the Creative Commons Attribution (CC-BY) license (http://creativecommons.org/licenses/by/4.0/). 